\title{
The chord length distribution of a dumbbell shaped aggregate:
}

\section{Analytical expression}

Frédéric Gruy ${ }^{\mathrm{a}, *}$ and Soong-Hyuck Suh ${ }^{\mathrm{b}}$

a Ecole Nationale Supérieure des Mines, 158 Cours Fauriel 42023 Saint-Etienne, France

b Department of Chemical Engineering, Keimyung University, Daegu, 704-701, Korea

\begin{abstract}
Dumbbell shaped aggregates are small particles synthesised in precipitation reactors. Their characterization by optical methods needs the chord length distribution (CLD) of such a shape. We present in this paper the analytical calculation of two CLD's corresponding to two different definitions of CLD. Comparison with Monte-Carlo simulations is presented. Good agreement is found between the exact calculation and simulations.
\end{abstract}

Keywords: Chord Length Distribution (CLD), Dumbbell, Aggregation, Anomalous Diffraction

\section{Introduction}

Many manufacturers use solid micro-particles in suspension for various applications: ceramics, paintings, pharmaceutics, cosmetics, food and chemicals. Particle sizing can be performed by physical methods based on the scattering between the particles and an incident electromagnetic wave. The scattered wave is depending on the particle morphology and on

Corresponding author.

E-mail address : fgruy@emse.fr (F. Gruy) 
the ratio between the refractive indices of particle material and suspending medium. Depending on the particle material and the selected method the measured signal may be straightforwardly related to the chord length distribution (CLD) of the randomly orientated particles set. This is illustrated by three examples:

- Small-Angle Scattering (SAS) measurements [1] are suitable for nano- and microparticles interacting with $\mathrm{X}$ rays. This method can be extended to optically soft microparticles interacting with light [2].

- Focused Beam Reflectance Measurements (FBRM) are among the most widely techniques for particle sizing [3]. It uses a focused beam of laser light that scans across particles passing in front of the probe window to measure a chord length distribution. The interpretation of the signal is only based on the reflected light. This method is suitable for particle size higher than $5 \mu \mathrm{m}$.

- Spectral Turbidimetry, i.e extinction measurement, is an optical method to measure the light scattering or extinction cross section of particles. In the case of large microparticles $(>1 \mu \mathrm{m})$ and very small optical contrast, extinction or forward scattering can be explained in the framework of anomalous diffraction approximation [2]: then the scattering cross section is expressed as an integral including the particle CLD. Anomalous diffraction approximation was applied to a sphere, an infinitely long circular cylinder, a prism column, a hexagonal crystal of ice, ellipsoids and a finite cylinder [4].

Depending on the physical principles of measurement the definition of the CLD for a given object changes: 3D isotropic uniform flow of infinite straight lines in the case of SAS and turbidity measurements, or 2D isotropic uniform flow of infinite straight lines for each projected area in the case of FBRM. 
The CLD of convex and non-convex bodies has been studied from a mathematical point of view. Explicit expressions have been obtained for bounded 2D or 3D convex domains: disc, triangle, rectangle, regular polygon [5], sphere, hemisphere [6], cylinders of various cross sections [7, 8], spheroids, polyhedron [9]. Moreover, Aharonyan [10] obtained an explicit expression for the orientation-dependent CLD for any bounded convex body.

Non-convex bodies have paid less attention than convex ones. Mazzolo et al [11] discussed the CLD in the context of reactor physics. They show that some relations between lower moments of CLD and simple geometric properties as volume, surface, $\ldots$ of the body remain valid for non-convex bodies whereas higher CLD moments do not obey the simple relations valid for convex bodies. Gille [12] studied the CLD of an infinitely long circular hollow cylinder that is a special case of non convex body; the corresponding calculation is based on basic principles. Gille [13] also considered two parallel circular cylinders separated by a short distance and calculated the 3D-correlation function that is related to CLD. Vlasov [14] introduced the notion of signed chord distribution for convex and non-convex bodies. He started from the work of Dirac transforming the six-dimensional integral of pairwise interaction potential for a convex body into a simpler expression including the CLD; he extended it to a non convex body. He showed that the expression of the integral is much more complicated than the one for convex body: it can be decomposed into several terms (integrals), each one related to the various segments of the given chord inside the non convex body. He formally deduces the expression of the CLD for the non-convex case.

Among the particle shapes observed in industrial processes, small clusters of spherical particles are often present. By the past we calculated [15] the CLD for a two-sphere aggregate. In this paper we extend this calculation to a set of two spheres penetrating each other. For instance, these dumbbell-like particles appear in the precipitation of inorganic 
compounds performed at high supersaturation and weak aggregation conditions. Polymeric colloids with such a morphology are also synthesised [16].

The section two of this paper develops a methodology in order to calculate the CLD of a dumbbell shaped aggregate. It is followed by a comparison with Monte Carlo Simulations in the section three. Section four is devoted to concluding remarks.

\section{Calculation of the chord length distribution}

A straight line may intersect more than one time across a non convex body. As a consequence, two CLD can be defined:

- The multiple chord distribution (MCD) where each segment interval on the same line is considered as one chord length separately. FBRM measurements are associated to MCD.

- The one chord distribution (OCD) where the sum of chord lengths for all intersected intervals is used as the definition of the chord length. SAS and turbidity measurements are associated to OCD.

Even if the latter ones only consider OCD, we will present both OCD and MCD calculations with $3 \mathrm{D}$ uniform flow of lines. The corresponding procedure is similar to the one used for a two-sphere aggregate [15].

Throughout the paper, the chord length distribution (density) is written $D(l) . D(l) d l$ is the number of chords within the $l$-range $[l, l+d l] . D(l)$ is normalized, i.e $\int_{0}^{l_{\max }} D(l) d l=1$.

\subsection{Definition of the different geometrical areas}


In the following of the paper, points will be denoted by lower-case letter (except the origin $\mathrm{O}$ of the coordinates system), line by upper-case letter, area by upper-case letter within parentheses and a volume by upper-case letter within brackets.

The dumbbell projection on a plane is considered. $\theta(0 \leq \theta \leq \pi / 2)$ is the angle between the line binding the centres of the two spheres (radius value equal to one) and the projection plane (coordinates $x, y$ ). The distance between the two sphere centres is denoted $\delta$. The centre of one of the two spheres is chosen as the origin $\mathrm{O}$ of the coordinates system (Fig. 1). The projection of the dumbbell is represented in the figures $2 \mathrm{a}-\mathrm{b}$ for two values of the $\theta$ angle and for a semiplane. The circles $\mathrm{C} 1$ and $\mathrm{C} 2$ represent the projection of the spheres (disks $(\mathrm{C} 1)$ and $(\mathrm{C} 2)$ ) whereas the dashed curve (ellipse later called E1 in the paper) represents the projection of the circular junction $\mathrm{J}$ between the two spheres. Let us consider a chord (perpendicular to the projection plane). According to the location of its intersection $(\mathrm{x}, \mathrm{y})$ point with the plane this line may cross:

- one single sphere: the point belongs to (P1)

- the junction between the two spheres: the point belongs to (PJ)

- or successively the two spheres: the point belongs to (P12).

The (Pf) area that is bounded by $\mathrm{C} 2$ (and $\mathrm{C} 1$ ) and E1 is fictitious. Its definition will be detailed hereafter.

The previously defined areas correspond to a full plane.

The ellipse E1 has some interesting properties:

- the coordinates of E1 centre are $(2 \delta \cos (\theta), 0)$

- the ellipse intersects the circles at the two b1 and b2 points (in fact four points if one considers the other semi-plane). The coordinates of the b2 point are 
$\left(\delta /(2 \cos (\theta)),\left(1-\delta^{2} /\left(4 \cos ^{2}(\theta)\right)\right)^{1 / 2}\right)$. The tangent at this point is common to the circle and the ellipse.

- as the $\theta$-angle increases $\mathrm{E} 1$ tends to a circle. $\mathrm{E} 1$ does not intersect $\mathrm{C} 1$ and $\mathrm{C} 2$ for $\theta>\theta_{E 1}=a \cos (\delta / 2)$

- the equation of the E1 ellipse is:

$(x-\delta \cos (\theta) / 2)^{2} / \sin ^{2}(\theta)+y^{2}=1-\delta^{2} / 4$

- $\mathrm{E} 1$ and $\mathrm{C} 2$ intersect the horizontal axis at $\mathrm{b} 1 *$ and $\mathrm{b} 1 * *$ respectively. The area defined by $\mathrm{b} 1, \mathrm{~b} 1 *$ and $\mathrm{b} 1 * *$ is the one-fourth of $(\mathrm{Pf})$.

\subsection{Decomposition of the CLD}

The CLD may be written as a sum of several partial CLD's.

\subsubsection{Calculation of the CLD (OCD)}

The chord length distribution of the dumbbell consists of the contributions of the various areas:

$$
\begin{aligned}
D^{O C D}(l)= & D_{P 12}(l)+D_{P J}(l)+D_{C 1+C 2}(l) \\
& -2 D_{C 1, P J}(l)-2 D_{C 1, P 12}(l)-2 D_{C 1, P f}(l)-2 D_{C 1, C 1 \cap C 2}(l)
\end{aligned}
$$

$D_{X}(l)$ is the CLD for the chords perpendicularly crossing the $(X)$ projected area. $D_{C 1, X}(l)$ is the contribution of the $(X)$ area to the CLD of a single sphere.

$D_{C 1, P J}, D_{C 1, P 12}, D_{C 1, P f}$ are calculated for $\theta<\theta_{E 1}$ whereas $D_{C 1, C 1 \cap C 2}$ is calculated for $\theta>\theta_{E 1}$. These partial distributions are equal to 0 outside the previously defined range of $\theta$-value.

\subsubsection{Calculation of the CLD (MCD)}


The chord length distribution of the dumbbell consists of the contributions of the various areas:

$D^{M C D}(l)=D_{P J}(l)+D_{C 1+C 2}(l)-2 D_{C 1, P J}(l)-2 D_{C 1, P f}(l)$

$D_{C 1, P f}$ is calculated for $\theta<\theta_{E 1}$ whereas $D_{C 1, P J}$ is calculated for any $\theta$.

In the following of the paper, the various contributions are calculated.

As the number of chords perpendicularly crossing a projection plane and the particle with a chord length within the range $[l, l+d l]$ is proportional to the corresponding projected area element, the relation between the projected area $S_{X}$ and the chord length distribution $D_{X}(l)$ is:

$D_{X}(l)= \pm N \frac{d S_{X}}{d l}$

$N$ is a normalization factor which will be specified later. Depending on the calculation, the area $S_{X}$ is the part of the projected area connected with a chord length smaller or higher than a given $l$ value. The equation 4 is integrated over the solid angle domain.

The calculation of $D_{X}(l)$ depends at once on the $\delta$ and $l$ values. For instance, certain $l$ values are not available for a given $\delta$ value; moreover, the shapes of (P1), (PJ), (P12), (Pf) projected areas depend on the $\delta$ values. As a consequence, a careful analysis of the various projected areas has to be performed before calculating the CLD's.

\subsection{Analysis of the various projected areas}

\subsubsection{Description of area (P12) and the corresponding chord length}

The length of the chord $(l)$ which crosses the two spheres obeys the equations:

$l=2\left(1-d^{2}\right)^{1 / 2}+2\left(1-d^{\prime 2}\right)^{1 / 2}$ if $\quad d<1, d^{\prime}<1$ 
with

$d=\left(x^{2}+y^{2}\right)^{1 / 2} \quad$ and $\quad d^{\prime}=\left((x-\delta \cos (\theta))^{2}+y^{2}\right)^{1 / 2}$

The locus of the points $(x, y)$ with constant $l$ is a segment of the E2 ellipse:

$\frac{(x-\delta \cos (\theta) / 2)^{2}}{a^{2}}+\frac{y^{2}}{b^{2}}=1 \quad|x-\delta \cos (\theta) / 2|<2 T /(\delta \cos (\theta))$

with

$a=\left[\frac{1-\delta^{2} \cos ^{2}(\theta) / 4-T}{1+T^{-1} \delta^{2} \cos ^{2}(\theta) / 4}\right]^{1 / 2}, \quad b=\left[1-\delta^{2} \cos ^{2}(\theta) / 4-T\right]^{1 / 2}$ and $\quad T=l^{2} / 16$

The dotted curve in the figure 3 a represents the ellipse E2. For small value of $l(l \square 1)$ the endpoint of $\mathrm{E} 2$ is close to the intersection point between $\mathrm{C} 1$ and $\mathrm{C} 2$. As the $l$ value increases the endpoint of E2 is going away from this intersection point. E1 and E2 are matching for a particular value of $l(=2 \delta \sin (\theta))$.

The ellipse E2 exists if $\left(b^{2}>0\right)$ :

$-\cos (\theta)<\cos \left(\theta_{0}\right)=2(1-T)^{1 / 2} / \delta$ and $T>1-\delta^{2} / 4$

or

$-T<1-\delta^{2} / 4$

The intersection of the ellipse $\mathrm{E} 2$ with the circle $\mathrm{C} 1$ exists if $A<1$ with

$A=\frac{2 T}{\delta \cos (\theta)}+\frac{\delta}{2} \cos (\theta)$

The coordinates of the intersecting point are: $x_{2}=A$ and $y_{2}=\left(1-x_{2}^{2}\right)^{1 / 2}$.

If $A>1$, there is no intersection (figure $3 b$ ).

One defines the angles $\theta_{+}$and $\theta_{-}$as:

$\cos \left(\theta_{ \pm}\right)=X_{ \pm}=\left(1 \pm(1-4 T)^{1 / 2}\right) / \delta$ 
which are the roots of $A=1$ ( if $l<2$ ).

The two angles $\theta_{ \pm}$exist if $0 \leq X_{ \pm} \leq 1$. In fact the conditions are more strict: $0 \leq X_{ \pm} \leq \cos \left(\theta_{E 1 E 2}\right)$. As only the part of E2 into the region bounded by $\mathrm{C} 1, \mathrm{C} 2$ and $\mathrm{E} 1$ is relevant, then the condition on $\theta$ is $l \leq 2 \delta \sin (\theta)$ i.e. $\sin (\theta) \geq \sin \left(\theta_{E 1 E 2}\right)=\left(4 T / \delta^{2}\right)^{1 / 2}$. It can be proved that $\theta_{E 1 E 2}>\theta_{0}$. As $\sin \left(\theta_{E 1 E 2}\right) \leq 1$, then $l \leq 2 \delta$. We emphasize that a chord goes across the two spheres if $\theta_{E 1 E 2}<\theta<\pi / 2$. This leads to an additional condition on $T$ and $\delta$.

So, the figure 4 defines the different areas in the $\left(\delta^{2} / 4, T\right)$-space: Each one corresponds to a specific configuration concerning C1, C2, E1 and E2. Each area also corresponds to a specific expression of the CLD.

Area 1: intersection between $\mathrm{C} 1$ and E2; $\nexists \theta_{+}, \exists \theta_{-} ; \theta_{E 1 E 2}<\theta_{-}<\pi / 2$

Area 2: no intersection between $\mathrm{C} 1$ and $\mathrm{E} 2: \nexists \theta_{ \pm}$

Area 3: intersection between $\mathrm{C} 1$ and $\mathrm{E} 2 ; \exists \theta_{ \pm} ; 0<\theta_{E 1 E 2}<\theta_{+}<\theta_{-}<\pi / 2$.

\subsubsection{Description of area $(\mathbf{P J})$ and the corresponding chord length}

$(\mathrm{PJ})$ area behaves as the (P12) area.

The length of the chord $(l)$ which crosses the two spheres obeys the equations:

$l=\left(1-d^{2}\right)^{1 / 2}+\left(1-d^{\prime 2}\right)^{1 / 2}+\delta \sin (\theta) \quad$ if $\quad d<1, d^{\prime}<1$

with

$d=\left(x^{2}+y^{2}\right)^{1 / 2} \quad$ and $\quad d^{\prime}=\left((x-\delta \cos (\theta))^{2}+y^{2}\right)^{1 / 2}$

The locus of the points $(x, y)$ with constant $l$ is the E3 ellipse:

$\frac{(x-\delta \cos (\theta) / 2)^{2}}{a^{\prime 2}}+\frac{y^{2}}{b^{\prime 2}}=1$ 
with

$$
\begin{aligned}
& a^{\prime}=\left(4 T^{1 / 2}-\sin (\theta)\right)\left[1-\delta^{2} / 4-4 T+2 \delta T^{1 / 2} \sin (\theta)\right]^{1 / 2} /\left[\delta^{2} / 4+4 T-2 \delta T^{1 / 2} \sin (\theta)\right]^{1 / 2} / 2 \\
& b^{\prime}=\left[1-\delta^{2} / 4-4 T+2 \delta T^{1 / 2} \sin (\theta)\right]^{1 / 2}
\end{aligned}
$$

The figure 5 represents the E3 ellipse (dotted line). The properties of E3 are the following:

- $\quad$ E3 corresponds to the inequality $l \geq 2 \delta \sin (\theta)$

- $\quad$ E3 exists for any $\theta$ if $4 T<1-\delta^{2} / 4$

- $\quad$ E3 exists for $\theta \geq \theta_{2}$ with $\sin \left(\theta_{2}\right)=\left(l^{2}+\delta^{2}-4\right) /(2 \delta l)$ if $4 T>1-\delta^{2} / 4$

- $\quad \mathrm{E} 3=\mathrm{E} 1$ for $l=2 \delta \sin (\theta)$

- E3 may intersect E1. The $\mathrm{X}$-coordinate of the intersection point is: $x_{13}=\delta \cos (\theta) / 2 \pm \tan (\theta)(l-\delta \sin (\theta)) / 2$

- $\mathrm{E} 3 \quad$ intersects $\quad \mathrm{E} 1 \quad$ if $\quad \tan (\theta)(l-\delta \sin (\theta)) / 2 \leq \sin (\theta)\left(1-\delta^{2} / 4\right)^{1 / 2}, \quad$ i.e. $B=l^{2}+\delta^{2}-2 l \delta \sin (\theta)-4 \cos ^{2}(\theta) \leq 0$. Then one can define two angles $\theta^{+}, \theta^{-}$:

$\sin \left(\theta^{ \pm}\right)=X^{ \pm}=\delta T^{1 / 2} \pm\left(1+\delta^{2} T-4 T-\delta^{2} / 4\right)^{1 / 2}$

which are the roots of $B=0$ ( if $l<2$ ).

The two angles $\theta^{ \pm}$exist if $0 \leq X^{ \pm} \leq 1$. In fact the inequalities are more strict: $\sin \left(\theta_{2}\right) \leq X^{ \pm} \leq \sin \left(\theta_{E 1 E 2}\right)$. This leads to an additional condition on $T$ and $\delta$.

So, the figure 6 defines the different areas in the $\left(\delta^{2} / 4, T\right)$-space: Each one corresponds to a specific configuration concerning E1 and E3. Each area also corresponds to a specific expression of the CLD.

Area 1a: no intersection between E1 and E3; $\nexists \theta^{+}, \nexists \theta^{-}, \theta_{2}=0$ 
Area 1b: intersection between E1 and E3; $\nexists \theta^{+}, \exists \theta^{-}, \theta_{2}>0 ; \theta_{2}<\theta^{-}<\theta_{E 1 E 2}$

Area 2a: intersection between E1 and E3; $\exists \theta^{+}, \nexists \theta^{-}, \theta_{2}=0 ; \theta_{2}<\theta^{+}<\theta_{E 1 E 2}$

Area 2b: intersection between E1 and E3; $\exists \theta^{ \pm}, \theta_{2}>0 ; 0<\theta_{2}<\theta^{-}<\theta^{+}<\theta_{E 1 E 2}$

Area 3: intersection between E1 and E3: $\nexists \theta^{ \pm}, \theta_{2}>0$

Area 4: no intersection between E1 and E3: $\nexists \theta^{ \pm}, \theta_{2}>0$

As only the part of E3 inside E1 is relevant, then the condition on $\theta$ is $l \geq 2 \delta \sin (\theta)$ i.e. $\sin (\theta) \leq \sin \left(\theta_{E 1 E 2}\right)=\left(4 T / \delta^{2}\right)^{1 / 2}$. As $\sin \left(\theta_{E 1 E 2}\right) \leq 1$, then $l \leq 2 \delta$

\subsubsection{Description of area (P1) and the corresponding chord length}

Area (P1) corresponds to the path of a line (chord) across a single sphere. The simplest way to calculate the corresponding CLD is to consider the various contributions of each projection area knowing that $(P 1)+(P J)+(P 12)+(P f)=(C 1)=(C 2)$. However two cases appear:

- E1 is tangential to the circles $\mathrm{C} 1$ and $\mathrm{C} 2$ : we have to calculate the contribution of (PJ), (P12) and (Pf) zones.

- $\quad \mathrm{E} 1$ is not tangential to the circles $\mathrm{C} 1$ and $\mathrm{C} 2:(P 1)+(C 1) \cap(C 2)=(C 1)=(C 2)$. Note that $(P J)+(P 12) \neq(C 1) \cap(C 2)$.

The transition between these two cases happens for $\cos (\theta)=\delta / 2\left(\theta=\theta_{E 1}\right)$.

\section{Contribution of $(\mathbf{C 1}) \cap(\mathrm{C2})\left(\theta>\theta_{E 1}\right)$}

The figure 7 describes the different areas in the $\left(\delta^{2} / 4, T\right)$-space: each one corresponds to a specific configuration concerning $\mathrm{C} 1$ and $\mathrm{C} 2$. Each area also corresponds to a specific expression of the CLD. Each area corresponds to a set of inequalities: 
$\delta>1$ or $\delta<1$

$(l / 2)^{2}+(\delta-1)^{2}>1$ or $(l / 2)^{2}+(\delta-1)^{2}<1$

$\cos \left(\theta_{ \pm}\right)=\left(1 \pm(1-4 T)^{1 / 2}\right) / \delta \leq \cos \theta_{E 1}=\delta / 2$

$\theta_{ \pm}$is such as $(l / 2)^{2}+(\delta \cos (\theta)-1)^{2}=1$, i.e. $A=1$

Each area is characterized by several angular intervals $\left[\theta_{j} ; \theta_{j+1}\right]_{k} \quad(j=1,2)$ defined as:

Area 1: $\left[\theta_{E 1} ; \theta_{-}\right]_{1}\left[\theta_{-} ; \pi / 2\right]_{2}$

Area 2: $\left[\theta_{E 1} ; \pi / 2\right]_{2}$

Area 3: $\left[\theta_{+} ; \theta_{-}\right]_{1}\left[\theta_{-} ; \pi / 2\right]_{2}$

The index $k$ corresponds to the angular distance $\Delta_{k} \psi$ :

1: $\Delta_{1} \psi=\varphi$

2: $\Delta_{2} \psi=\pi$

Where $\varphi=\operatorname{acos} \frac{\delta \cos (\theta) / 2-2 T /(\delta \cos (\theta))}{\left(1-l^{2} / 4\right)^{1 / 2}}$

\section{Contribution of $(\boldsymbol{P J})$ (for any $\theta$ )}

We are looking for the intersection between E1 and a circle denoted C3 with radius $\left(1-l^{2} / 4\right)^{1 / 2}(l$ is constant). Depending on $\theta$-value, 0,1 or 2 intersection points are present. In case of 2 intersection points, their $x$-coordinates are $u_{ \pm}=\frac{\delta \pm l \sin (\theta)}{2 \cos (\theta)}$.

The figure 8 is a diagram in the $(\theta, x)$ space showing the intersection of $\mathrm{C} 3$ with $\mathrm{E} 1$. 
Each line segment of the dash-dot curve corresponds to an angle interval with 0,1 or 2 intersection points. We called $\theta_{+}^{l}, \theta_{+}^{r}, \theta_{-}^{l}, \theta_{-}^{r}$ the boundary angles between segments (r: right branch; 1: left branch; +: solid line; -: dashed line).

Depending on $(\delta, l)$ values, one identifies several areas in the $\left(\delta^{2} / 4, T\right)$ diagram (figure 9$)$.

Each area has three angular intervals $\left[\theta_{j} ; \theta_{j+1}\right]_{k} \quad(j=1,2,3)$ defined as:

Area 1: $\left[0 ; \theta_{+}^{l}\right]_{2} \quad\left[\theta_{+}^{l} ; \theta_{+}^{r}\right]_{1}\left[\theta_{+}^{r} ; \pi / 2\right]_{0}$

Area 2: $\left[0 ; \theta_{-}^{l}\right]_{0} \quad\left[\theta_{-}^{l} ; \theta_{-}^{r}\right]_{1}\left[\theta_{-}^{r} ; \pi / 2\right]_{3}$

Area $3:\left[0 ; \theta_{-}^{l}\right]_{0}\left[\theta_{-}^{l} ; \theta_{+}^{r}\right]_{1}\left[\theta_{+}^{r} ; \pi / 2\right]_{0}$

Area 4: $\left[0 ; \theta_{+}^{l}\right]_{2} \quad\left[\theta_{+}^{l} ; \theta_{-}^{r}\right]_{1}\left[\theta_{-}^{r} ; \pi / 2\right]_{3}$

The index $k$ corresponds to the angular distance $\Delta_{k} \psi$ :

$0: \Delta_{0} \psi=0$

$1: \Delta_{1} \psi=\psi_{-}$

2: $\Delta_{2} \psi=\psi_{-}-\psi_{+}$

3: $\Delta_{3} \psi=\pi$

Where $\psi_{ \pm}=a \cos \left(u_{ \pm} /\left(1-l^{2} / 4\right)^{1 / 2}\right)$

\section{Contribution of (PJ) $\left(\theta<\theta_{E 1}\right)$}

The various areas in the $\left(\delta^{2} / 4, T\right)$ sub-space are already described in the figure 6 .

Each area has several angular intervals $\left[\theta_{j} ; \theta_{j+1}\right]_{k}$ defined as:

Area 1a: $\left[0 ; \theta_{+}^{l}\right]_{2}\left[\theta_{+}^{l} ; \theta_{E 1}\right]_{1}$

Area $1 \mathrm{~b}:\left[0 ; \theta_{-}^{l}\right]_{0}\left[\theta_{-}^{l} ; \theta_{E 1}\right]_{1}$ 
Area 2a: $\left[0 ; \theta_{+}^{l}\right]_{2} \quad\left[\theta_{+}^{l} ; \theta_{-}^{r}\right]_{1} \quad\left[\theta_{-}^{r} ; \theta_{E 1}\right]_{3}$

Area $2 \mathrm{~b}:\left[0 ; \theta_{-}^{l}\right]_{0} \quad\left[\theta_{-}^{l} ; \theta_{-}^{r}\right]_{1}\left[\theta_{-}^{r} ; \theta_{E 1}\right]_{3}$

Area 3: $\left[0 ; \theta_{E 1}\right]_{0}$

Contribution of (P12) $\left(\theta<\theta_{E 1}\right)$

The allowed area for the calculation of the CLD is the area denoted 1 (figure 4).

Contribution of $(\boldsymbol{P f})\left(\theta<\theta_{E 1}\right)$

The allowed areas for the calculation of the CLD are the areas denoted 1a and 2a (figure 6).

\subsection{Contributions of the different areas to the CLD}

\subsubsection{Contribution of (P12) area to the CLD}

The fraction of the projected area, such as the chord length is smaller than $l$, obeys the equations:

- if $\mathrm{C} 1$ and $\mathrm{C} 2$ intersect E2

$S_{1}=4 \int_{0}^{x_{2}-\delta \cos \theta / 2}\left[\left(1-\left(x+\frac{\delta}{2} \cos (\theta)\right)^{2}\right)^{1 / 2}-b\left(1-x^{2} / a^{2}\right)^{1 / 2}\right] d x$

- if $\mathrm{C} 1$ and $\mathrm{C} 2$ do not intersect E2

$S_{2}=2(\alpha-\sin (2 \alpha))-\pi T^{1 / 2}\left[\left(T+\frac{\delta^{2}}{4} \cos ^{2}(\theta)\right)^{-1 / 2}-\left(T+\frac{\delta^{2}}{4} \cos ^{2}(\theta)\right)^{1 / 2}\right]$

With $\sin (\alpha)=\left(1-\frac{\delta^{2}}{4} \cos ^{2}(\theta)\right)^{1 / 2}$ 
The average CLD over all the orientations will be such as:

- area 1:

$$
D_{P 12}(l)=N \int_{\theta E 1 E 2}^{\theta_{\bar{C}}} d S_{1} / d l \cos (\theta) d \theta+N \int_{\theta_{-}}^{\pi / 2} d S_{2} / d l \cos (\theta) d \theta
$$

- area 2:

$$
D_{P 12}(l)=N \int_{\theta E 1 E 2}^{\pi / 2} d S_{2} / d l \cos (\theta) d \theta
$$

- area 3:

$$
D_{P 12}(l)=N \int_{\theta_{+}}^{\theta_{-}} d S_{1} / d l \cos (\theta) d \theta+N \int_{\theta E 1 E 2}^{\theta_{+}} d S_{2} / d l \cos (\theta) d \theta+N \int_{\theta_{-}}^{\pi / 2} d S_{2} / d l \cos (\theta) d \theta
$$

\subsubsection{Contribution of (PJ) area to the CLD}

The fraction of the projected area, such as the chord length is smaller than $l$, obeys the equations:

$$
\begin{aligned}
& S_{1}=4 \int_{0}^{x_{13}-\delta \cos (\theta) / 2}\left[\left(1-\delta^{2} / 4\right)^{1 / 2}\left(1-x^{2} / \sin ^{2}(\theta)\right)^{1 / 2}-b^{\prime}\left(1-x^{2} / a^{\prime 2}\right)^{1 / 2}\right] d x \\
& \quad-\text { if E3 does not intersect E1 } \\
& S_{2}=\pi\left(1-\delta^{2} / 4\right) \sin (\theta)-\pi a^{\prime} b^{\prime}
\end{aligned}
$$

The average CLD over all the orientations will be such as:

- area 1a:

$$
D_{P J}(l)=N \int_{0}^{\theta E 1 E 2} d S_{1} / d l \cos (\theta) d \theta
$$

- area $1 b$ : 


$$
D_{P J}(l)=N \int_{\theta^{-}}^{\theta E 1 E 2} d S_{1} / d l \cos (\theta) d \theta+N \int_{\theta 2}^{\theta^{-}} d S_{2} / d l \cos (\theta) d \theta
$$

- area 2a:

$$
D_{P J}(l)=N \int_{\theta^{+}}^{\theta E 1 E 2} d S_{2} / d l \cos (\theta) d \theta+N \int_{0}^{\theta^{+}} d S_{1} / d l \cos (\theta) d \theta
$$

area $2 b$ :

$$
D_{P J}(l)=N \int_{\theta^{-}}^{\theta^{+}} d S_{1} / d l \cos (\theta) d \theta+N \int_{\theta 2}^{\theta^{-}} d S_{2} / d l \cos (\theta) d \theta+N \int_{\theta^{+}}^{\theta E 1 E 2} d S_{2} / d l \cos (\theta) d \theta
$$

- $\quad$ areas 3 and 4:

$$
D_{P J}(l)=N \int_{\theta 2}^{\theta E 1 E 2} d S_{2} / d l \cos (\theta) d \theta
$$

If $l>2 \delta$ then $\theta_{E 1 E 2}=\pi / 2$

\subsubsection{Contribution of (P1) area to the CLD}

\section{Contribution of $(\mathbf{C 1}) \cap(\mathrm{C2})\left(\theta>\theta_{E 1}\right)$}

Let us consider the sphere of centre $\mathrm{O}$ and its projection. The area element $d S$ belonging to the intersection of the two disks (projected spheres) and having the chord length in the range $[l, l+d l]$, is:

$$
\begin{aligned}
& \text { - if } \cos (\theta)>1 / \delta d S_{\varphi}=r_{1} \varphi d r_{1} \quad \text { with } \quad r_{1} \in[(\delta \cos (\theta)-1), 1] \\
& \text { - if } \cos (\theta)<1 / \delta d S_{\pi}=r_{1} \pi d r_{1} \quad \text { with } \quad r_{1} \in[0,(1-\delta \cos (\theta))] \\
& d S_{\varphi}=r_{1} \varphi d r_{1} \quad \text { with } \quad r_{1} \in[(1-\delta \cos (\theta)), 1]
\end{aligned}
$$

With $r_{1}=\left(1-l^{2} / 4\right)^{1 / 2}=(1-4 T)^{1 / 2}$. 
The average (for the 2 spheres) over all the orientations is written:

- area 1:

$$
D_{C 1, C 1 \cap C 2}(l)=-N \int_{\theta E 1}^{\theta-} 2 d S_{\varphi} / d l \cos (\theta) d \theta-N \int_{\theta-}^{\pi / 2} 2 d S_{\pi} / d l \cos (\theta) d \theta
$$

- $\quad$ area 2:

$$
D_{C 1, C 1 \cap C 2}(l)=-N \int_{\theta E 1}^{\pi / 2} 2 d S_{\pi} / d l \cos (\theta) d \theta
$$

- area 3:

$$
D_{C 1, C 1 \cap C 2}(l)=-N \int_{\theta_{+}}^{\theta_{\overline{+}}} 2 d S_{\varphi} / d l \cos (\theta) d \theta-N \int_{\theta_{-}}^{\pi / 2} 2 d S_{\pi} / d l \cos (\theta) d \theta
$$

\section{Contribution of (PJ)}

$D_{C 1, P J}(l)=-N \sum_{j=1}^{3} \int_{\theta_{j}}^{\theta_{j+1}} 2 r_{1} \Delta_{k(j)} \psi\left(d r_{1} / d l\right) \cos (\theta) d \theta$

With $r_{1}=\left(1-l^{2} / 4\right)^{1 / 2}$

This relation is valid at once for $\left(\theta<\theta_{E 1}\right)$ and $\left(\theta<\theta_{E 1}\right.$ and $\left.\theta>\theta_{E 1}\right)$

\section{Contribution of (P12) $\left(\theta<\theta_{E 1}\right)$}

One considers the $d_{1} d_{2}$ arc (or the corresponding angle) defined by the two d 1 and $\mathrm{d} 2$ points: $\mathrm{d} 1$ is the intersection point of $\mathrm{C} 3$ and $\mathrm{E} 1, \mathrm{~d} 2$ is the intersection of $\mathrm{C} 3$ and $\mathrm{C} 2$.

$$
d_{1} d_{2}=\varphi-a \cos \left(u_{-} / r_{1}\right)
$$

The swept area by increasing the radius of the $\mathrm{C} 3$ circle is:

$$
\begin{aligned}
& d S=r_{1} d_{1} d_{2} d r_{1} \\
& D_{C 1, P 12}(l)=-N \int_{a \sin (l /(2 \delta))}^{\theta E 1} 2 d S / d l \cos (\theta) d \theta
\end{aligned}
$$


Contribution of $(\boldsymbol{P f})\left(\theta<\theta_{E 1}\right)$

The swept area by increasing the radius of the $\mathrm{C} 3$ circle is:

$$
\begin{aligned}
& d S=r_{1} \psi_{+} d r_{1} \\
& D_{C 1, P f}(l)=-N \int_{0}^{\theta_{+}^{l}} 2 d S / d l \cos (\theta) d \theta
\end{aligned}
$$

\section{Contribution of $(C 1)+(C 2)$}

The chord length distribution of the 2 spheres considered alone is obtained while taking $\varphi=\pi$ :

$D_{C 1+C 2}(l)=2 N \pi(l / 2)$

\section{Normalization factor}

The normalization factor $N$ is the inverse of the dumbbell projected area averaged over all the orientations:

$$
N^{-1}=\left\langle S_{p}\right\rangle_{\theta}=\int_{0}^{\pi / 2} S_{p} \cos (\theta) d \theta
$$

With

$$
S_{p}=2 \pi+\delta \cos (\theta)\left(1-\delta^{2} \cos ^{2}(\theta) / 4\right)^{1 / 2}-4 a \cos (\delta \cos (\theta) / 2)
$$

\section{Comparison with Monte Carlo Simulations}

Two procedures for Monte Carlo Simulations (MCS) have been used: 
i) A classical MC (Monte Carlo) software generates an isotropic uniform random line across the geometric object, and then collects the chord length segments. The successive steps of the algorithm are:

1. Choice of a line or the corresponding unit vector $\vec{n}$ with random orientation

2. Projection of the dumbbell on the plane perpendicular to the vector $\vec{n}$, i.e. determination of the coordinates of the centres of discs

3. Calculation of the intercept, i.e. length of the segment perpendicular to the plane and crossing the dumbbell

4. Go to step 1

The calculation of the intercept $I$ depends on the value of $Q_{i}$ given by $Q_{i}=1-\left(x-x_{i}\right)^{2}-\left(y-y_{i}\right)^{2}$ with $i=1,2$ and where $\left(x_{i}, y_{i}\right)$ are the coordinates of the centres of the projected spheres and $(x, y)$ are the coordinates of intersection of the line and the plane. Then,

If $Q_{1}<0$ and $Q_{2}<0$ then $I=0$

If $Q_{1}>0$ and $Q_{2}<0$ then $I=2 \sqrt{Q_{1}}$

If $Q_{1}<0$ and $Q_{2}>0$ then $I=2 \sqrt{Q_{2}}$

If $Q_{1}>0$ and $Q_{2}>0$ then

$$
\left\{\begin{array}{l}
d=\sqrt{Q_{1}}+\sqrt{Q_{2}}+\left(\delta^{2}-\left(x_{2}-x_{1}\right)^{2}-\left(y_{2}-y_{1}\right)^{2}\right)^{1 / 2} \\
D=2 \sqrt{Q_{1}}+2 \sqrt{Q_{2}} \\
S=\sup \left\{2 \sqrt{Q_{1}}, 2 \sqrt{Q_{2}}\right\}
\end{array}\right.
$$

If $D>d$ then $I=\sup \{S, d\}$

If $D<d$ then $I=D$ for $\mathrm{OCD}$

If $D<d$ then $I=2 \sqrt{Q_{1}}$ and $\mathrm{I}=2 \sqrt{Q_{2}}$ for $\mathrm{MCD}$ 
$2 \sqrt{Q_{i}}$ is the length of the chord in the sphere $i . d$ is the distance between the two end points of the line crossing the two spheres.

The MC sampling distribution may be visually represented as the discrete probability histogram. The chord length between zero and the maximal possible length is divided on mbins with the equal size of $\Delta \mathrm{l}$. All the simulation runs have been carried out by generating $10^{7}$ unbiased random lines. The sampling error is smaller than $10^{-4}$.

ii) An alternative algorithm is suitable for application in optics (AD approximation, see [15]). It completes the second step of the preceding algorithm by scanning the discretized projected area and by calculating the corresponding chord length for each point belonging to the grid. The other steps of the algorithm are not modified. If this sequence is repeated $10^{5}$ times, the sampling error is smaller than $10^{-3}$. The two algorithms are in very good agreement. However this variant will not be used in this work.

The analytical $\mathrm{CLD}^{1}$ (section 2) is compared to the data coming from MCS. Figure 11 shows such a comparison for $\delta=0.4$ (MCD). The agreement between the two methods is very good. Similar results are obtained for the two types of CLD (MCD and OCD) and for all $\delta$-values. Figure 12 represents the relative difference $\sigma$ between the two methods: ${ }^{\S}$

\footnotetext{
${ }^{\S}$ The software may be provided by simple request to the author.
} 
$\sigma=\left(D_{M C S}(l)-D(l)\right) / D(l)$

It can be observed that the relative deviation is very small except about $l=2$. This last feature is due to the sharp discontinuity for $D(l)$ at $l=2$ (Fig. 11). The range of $l$-value corresponding to this large deviation is $[1.98 ; 2.02]$. MCS and our analytical calculations (including numerical integration) fail together within this $l$-range. For comparing the two methods by a quantitative way we have calculated the standard relative deviation as:

$\Sigma=\left[\frac{1}{N} \sum_{i=1}^{N}\left(D_{M C S}\left(l_{i}\right) / D\left(l_{i}\right)-1\right)^{2}\right]^{1 / 2}$

The summation is performed with the chords in the ranges $[0 ; 1.98]$ and $[2.02 ; 4]$.

The $\Sigma$-value has been calculated for each $\delta$-value and for the two types of CLD. The $\Sigma$-value is always within the [0.002-0.004] range.

\section{Conclusion}

The CLD for the two types of chord length (OCD and MCD) has been analytically calculated. A good agreement was found between the analytical expression of the CLD and Monte-Carlo simulations. The analytical CLD for dumbbell-like aggregates is an illustration of the ideas of Vlasov [14]: non convexity leads to complicated expressions for CLD that are built by considering the contributions coming from several parts of the body. This work will be used for studying systematically the effect of the $\delta$ parameter on the CLD and its moments. It also permits to compare OCD and MCD. The corresponding results will be soon published. 


\section{References}

[1] A. Guinier and G. Fournet, Small-Angle scattering of X-rays, Wiley, New York, 1955

[2] H.C. Van de Hulst, Light scattering by small particles, John Wiley, NewYork, 1957.

[3] M. Li and D. Wilkinson, Chemical Engineering Science, 2005. 60: p. 3251-3265.

[4] A.A. Kokhanovsky, Optics of light scattering media, Springer, Berlin, 2001

[5] V.K. Ohanyan and N.G. Aharonyan "Tomography of bounded convex domains" International Journal of Mathematical Science Education 2(2009)1-12

[6] W. Gille, Computational Materials Science, 1999, 15: p. 449-454

[7] W. Gille "Chord length distributions of infinitely long geometric figures" Powder Technology 123(2002)292-298

[8] W. Gille "Chord length distribution density of a triangular rod" Computational Materials Science 22(2001)151-154

[9] H.S. Sukiasian "Three-dimensional Pleijel identity and its application" Izvestiya Natsionalnoi Akademii Nauk Armenii Matematika 38(2003)53-69

[10] N.G. Aharonyan and V.K. Ohanyan "Chord length distribution functions for polygons" J. of contemporary mathematical analysis 40(2005)43-56

[11] A. Mazzolo, B. Rooesslinger, C.M. Diop, "On the properties of the chord length distribution, from integral geometry to reactor physics", Annals of Nuclear Energy 30(2003)1391-1400

[12] W. Gille "Chord length distribution density of an infinitely long circular hollow cylinder" Mathematical and computer modelling 34(2001)423-431

[13] W. Gille "The small angle scattering correlation function of two infinitely long parallel circular cylinders" Computational Materials Science 20(2001)181-195

[14] A. Yu. Vlasov "Signed chord length distribution" arXiv:0711.4734v3 [math-ph] 17 Dec 2007 
[15] F. Gruy and S. Jacquier,"the chord length distribution of a two-sphere aggregate", Computational Materials Science 44(2008)218-223

[16] H.R. Vutukuri, A. Van Blaaderen and A. Imhof, " Synthesis of fluorescent monodisperse non-spherical dumbell-like model colloids”, J. Mater. Chem. 22(2012)21893-21900 


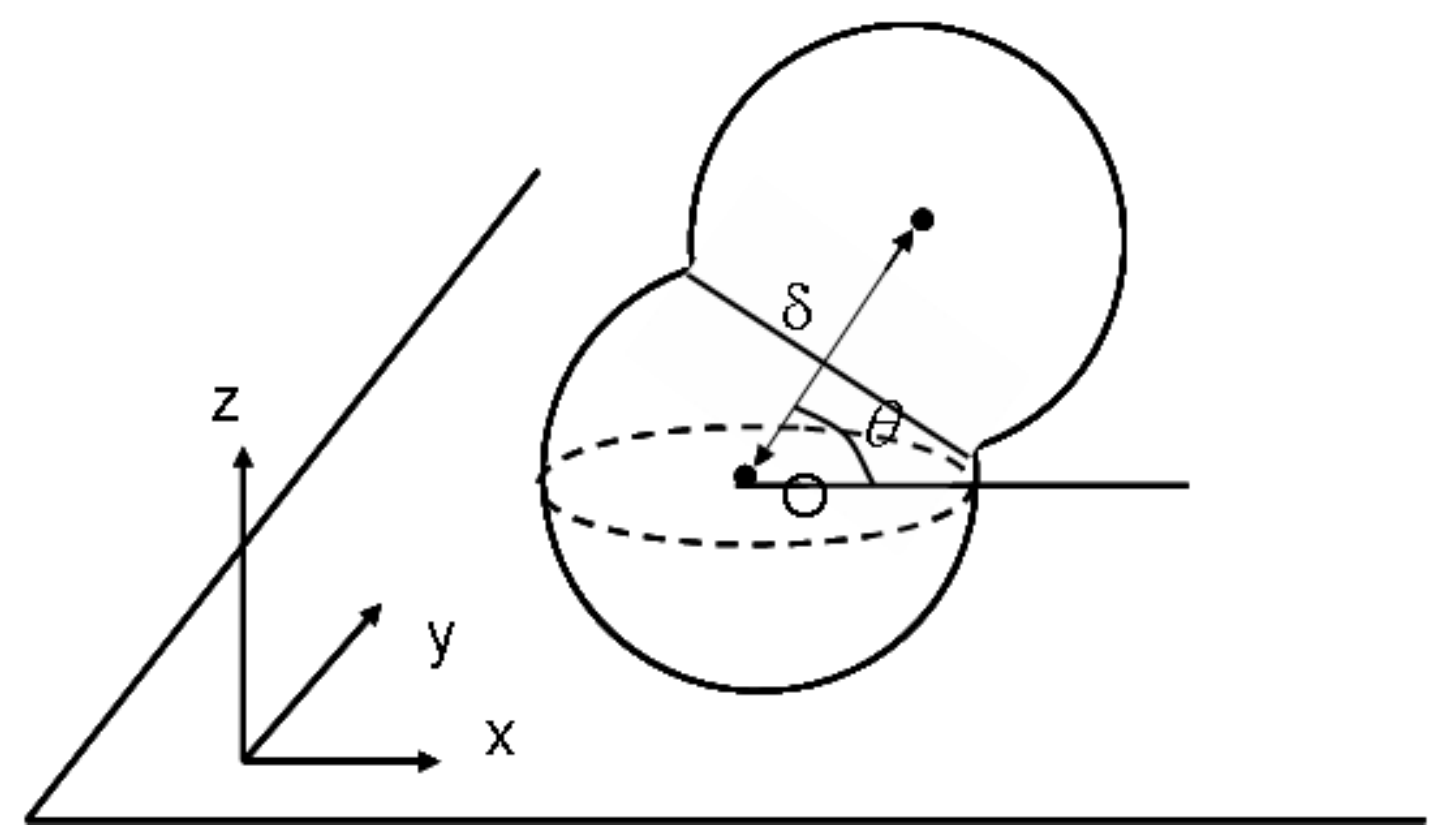

Figure 1: 3D drawing of the dumbbell shaped aggregate 


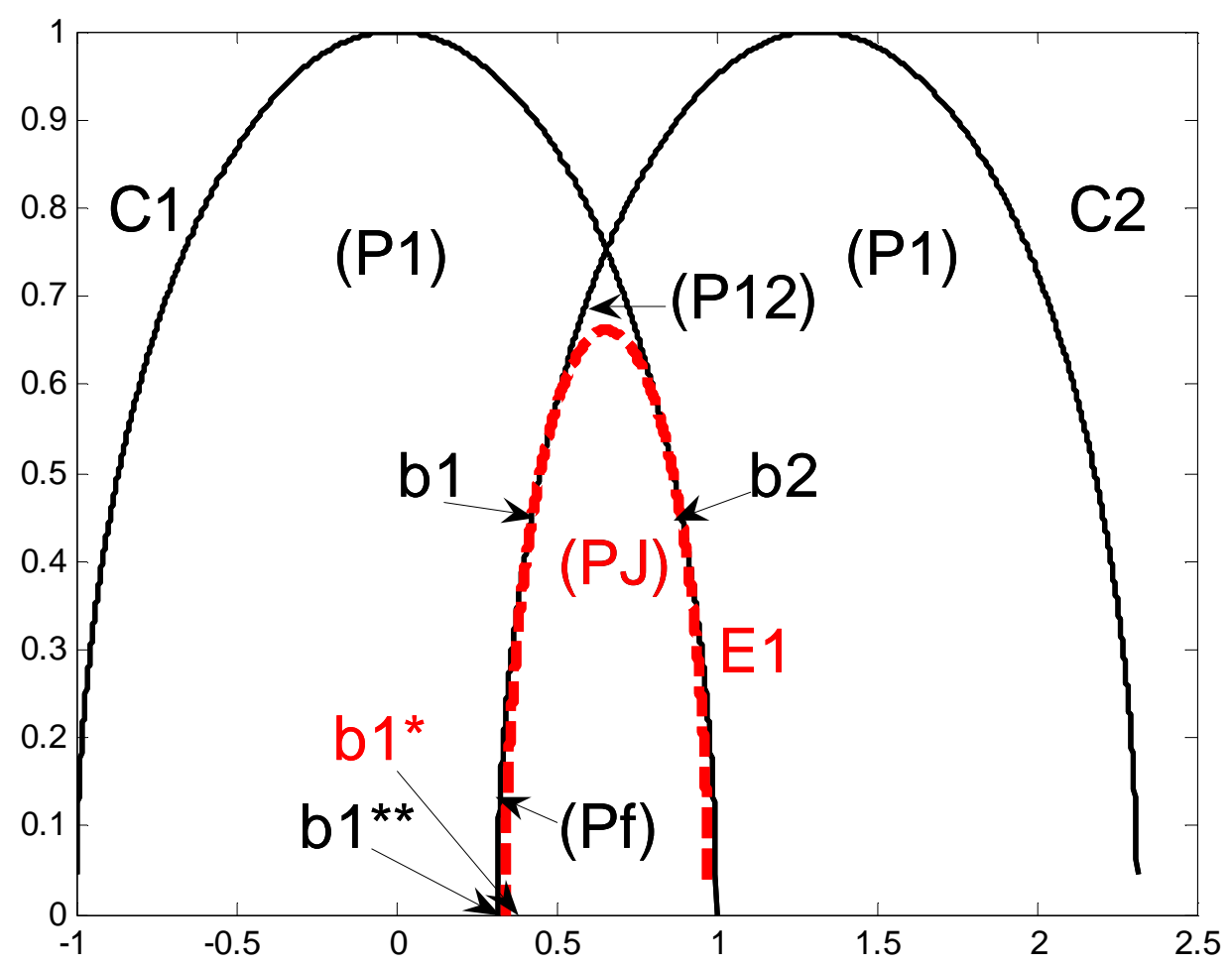

Figure 2a: projection $(\mathrm{x}, \mathrm{y})$ of the dumbbell shaped aggregate. $\delta=1.5$ and $\theta=0.5 \mathrm{rad}$. Circles $\mathrm{C} 1$ and C2: solid lines; ellipse E1: dashed line (red) 


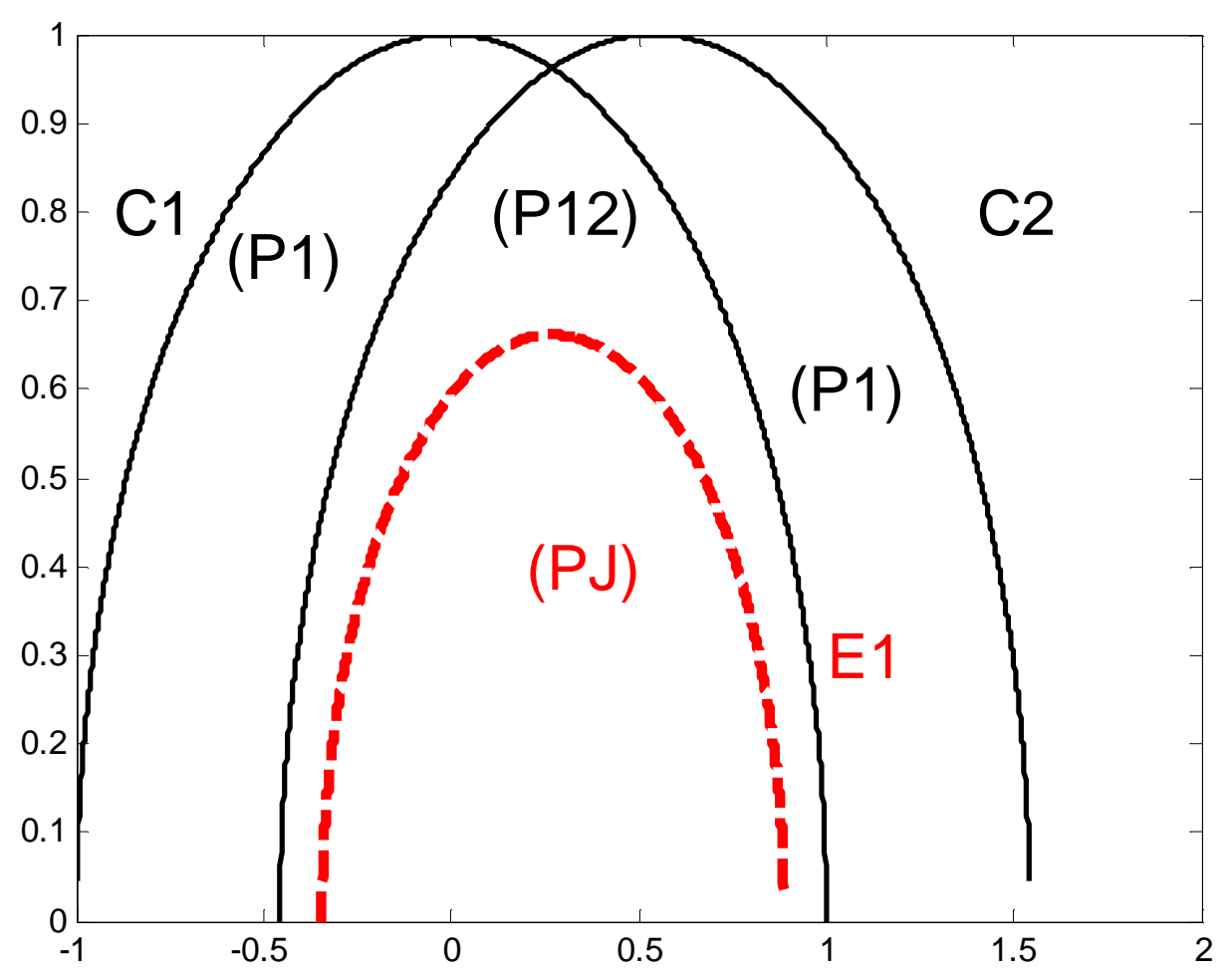

Figure 2b: projection $(\mathrm{x}, \mathrm{y})$ of the dumbbell shaped aggregate. $\delta=1.5$ and $\theta=1.2 \mathrm{rad}$. Circles $\mathrm{C} 1$ and C2: solid lines; ellipse E1: dashed line (red) 


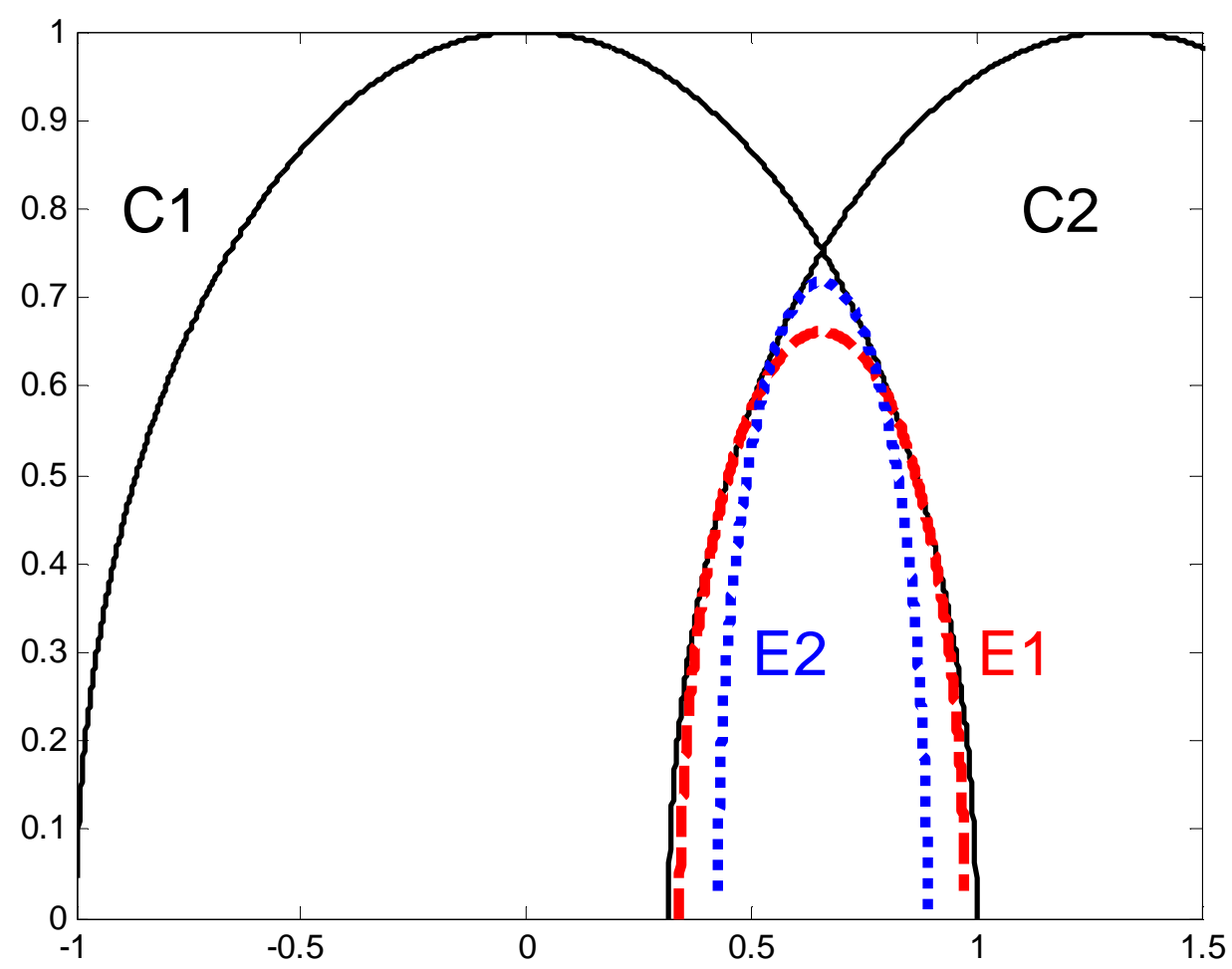

Figure 3a: projection $(\mathrm{x}, \mathrm{y})$ of the dumbbell shaped aggregate. $\delta=1.5$ and $\theta=0.5 \mathrm{rad} ; l=0.3 l_{\max }$ with $l_{\max }=4\left[1-\delta^{2} \cos ^{2}(\theta) / 4\right]^{1 / 2}$ Circles $\mathrm{C} 1$ and $\mathrm{C} 2$ : solid lines; ellipse E1: dashed line (red); ellipse E2: dotted line (blue) 


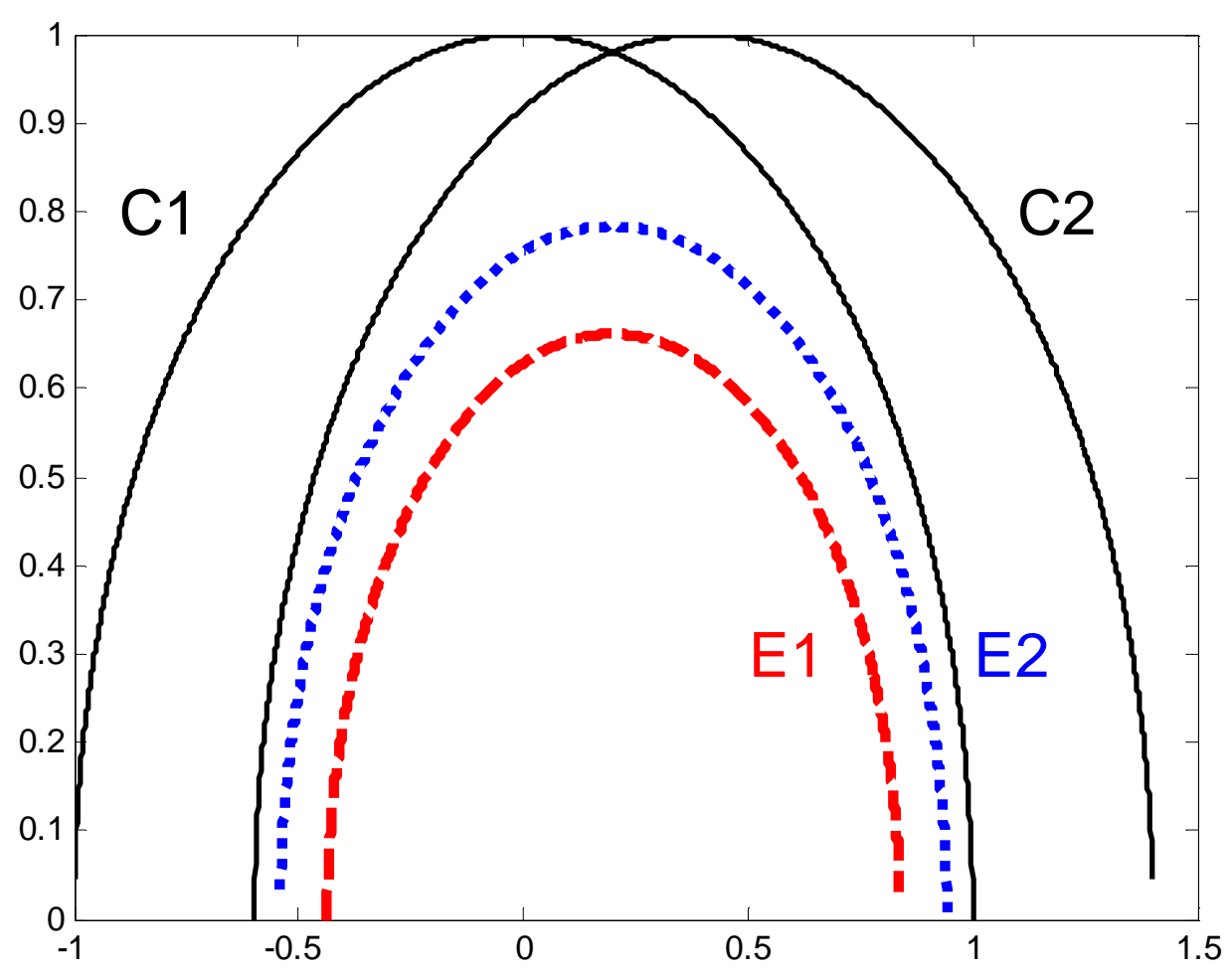

Figure 3b: projection $(\mathrm{x}, \mathrm{y})$ of the dumbbell shaped aggregate. $\delta=1.5$ and $\theta=1.3 \mathrm{rad} ; l=0.6 l_{\max }$ with $l_{\max }=4\left[1-\delta^{2} \cos ^{2}(\theta) / 4\right]^{1 / 2}$ Circles $\mathrm{C} 1$ and $\mathrm{C} 2$ : solid lines; ellipse E1: dashed line (red); ellipse E2: dotted line (blue) 


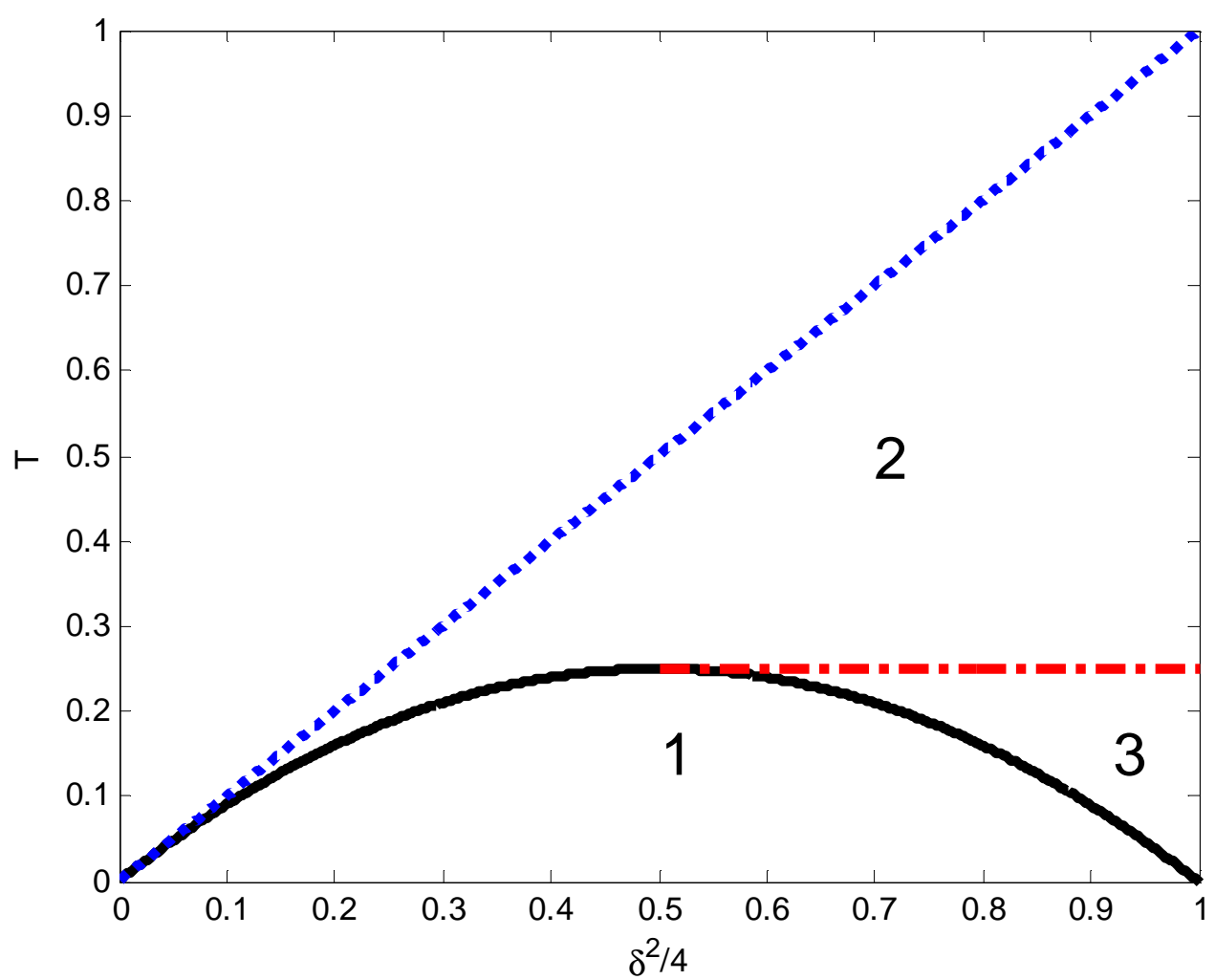

Figure 4: different areas of the $\left(\delta^{2} / 4, T\right)$ sub-space for a chord crossing the two spheres. The equations corresponding to the curves separating the different areas are:

Dash-dot line (Red): $T=1 / 4$; dotted line (blue): $T=\delta^{2} / 4 ;$ solid line (black): $T=\frac{\delta^{2}}{4}-\left(\frac{\delta^{2}}{4}\right)^{2}$ 


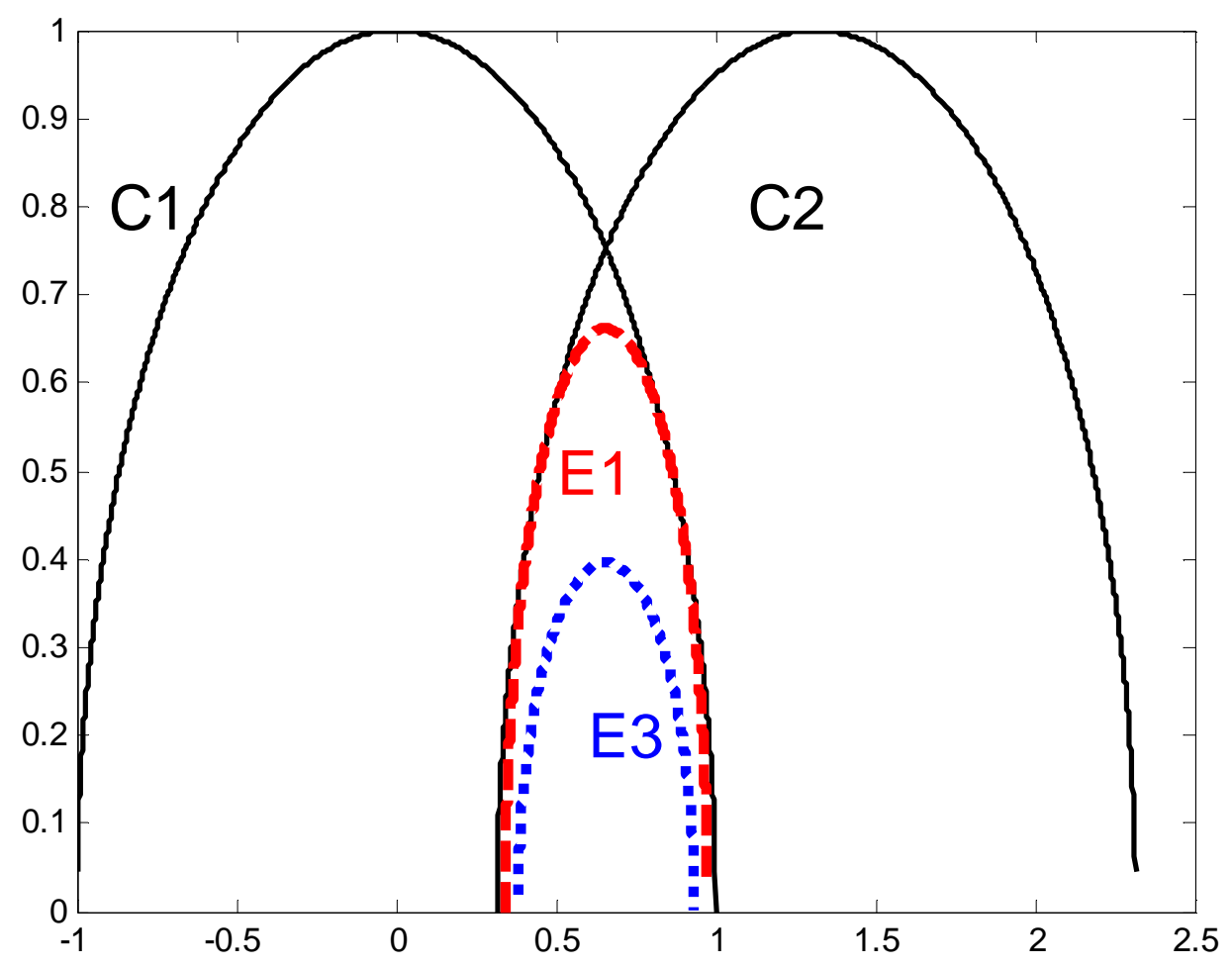

Figure 5: projection (x,y) of the dumbbell shaped aggregate. $\delta=1.5$ and $\theta=0.5 \mathrm{rad} ; 1=2$. Circles C1 and C2: solid lines; ellipse E1: dashed line (red); ellipse E3: dotted line (blue) 


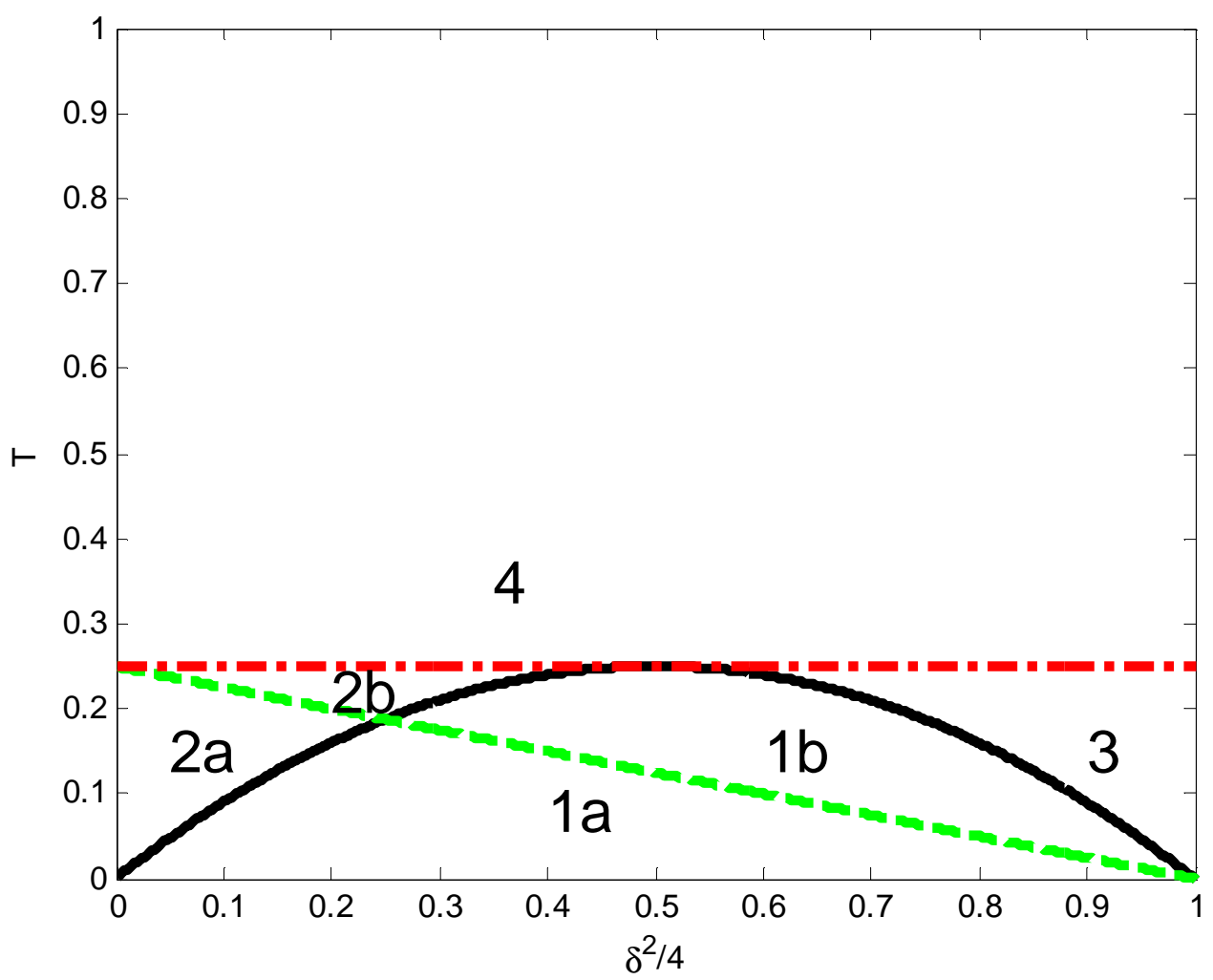

Figure 6: different areas of the $\left(\delta^{2} / 4, T\right)$ sub-space for a chord crossing the junction. The equations corresponding to the curves separating the different areas are:

Dashed line (green): $T=\left(1-\delta^{2} / 4\right) / 4$; dash-dot line (red): $T=1 / 4$; solid line (black) $T=\left(1-\frac{\delta^{2}}{4}\right) \frac{\delta^{2}}{4}$ 


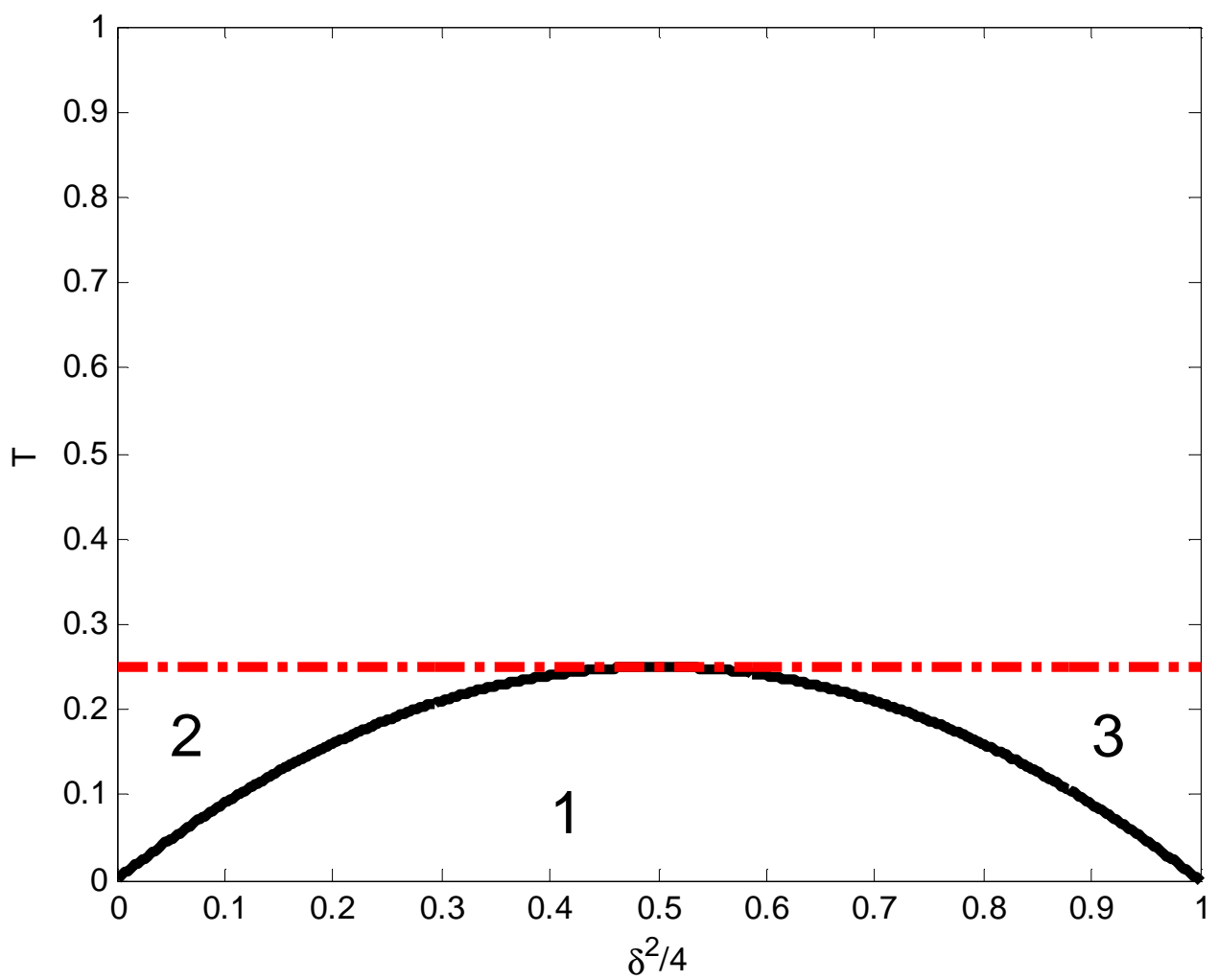

Figure 7: different areas of the $\left(\delta^{2} / 4, T\right)$ sub-space for a chord crossing a single sphere. The equations corresponding to the curves separating the different areas are:

Dash-dot line (red): $T=1 / 4$; solid line (black) $T=\left(1-\frac{\delta^{2}}{4}\right) \frac{\delta^{2}}{4}$ 


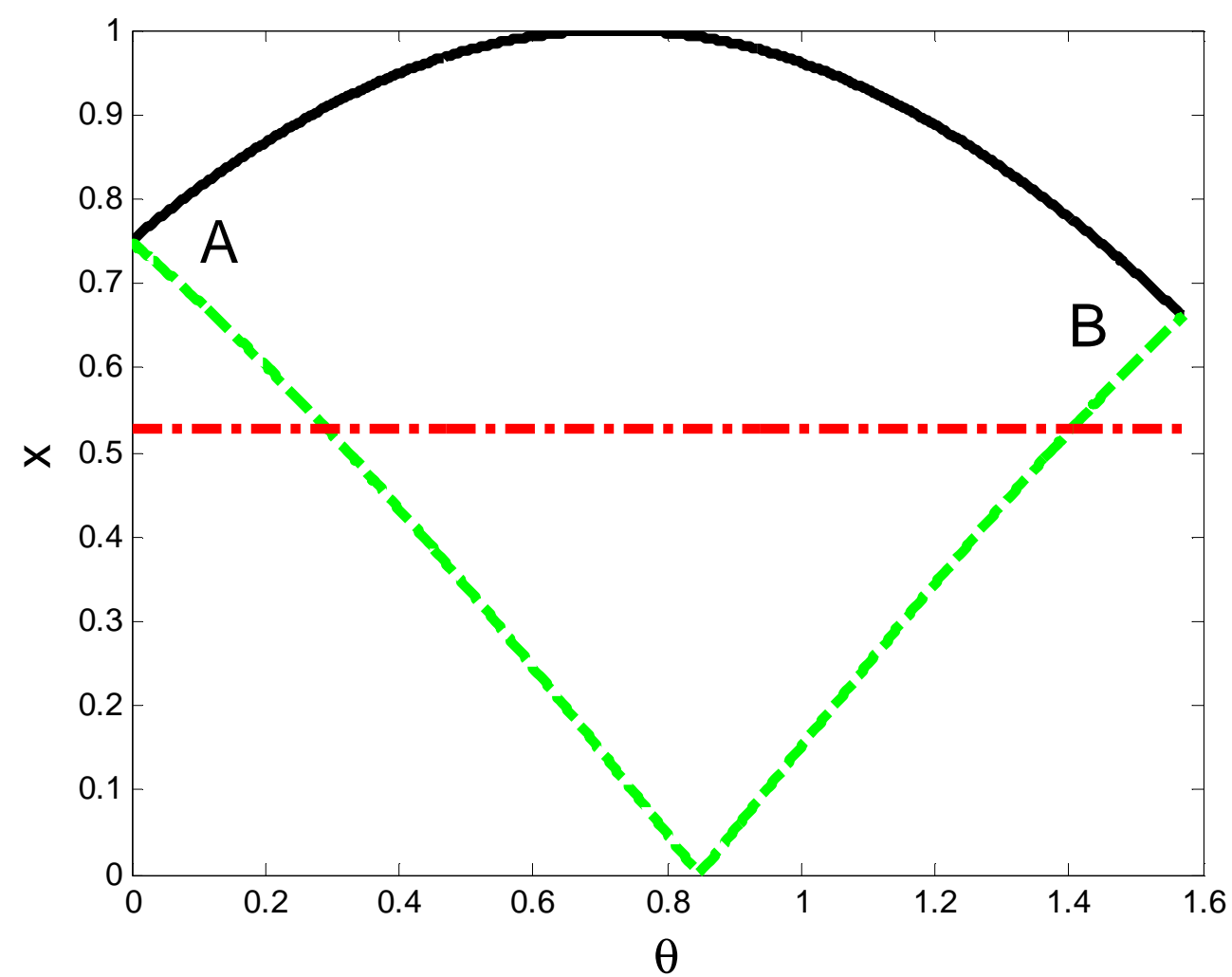

Figure 8: $(\theta, x)$ space for a chord crossing the sphere ((PJ) zone). The dash-dot line (red) corresponds to the radius of the $\mathrm{C} 3$ circle $\left(r=\left(1-l^{2} / 4\right)^{1 / 2}\right.$. The solid line (black) corresponds to the abscissa of the right endpoint of $\mathrm{E} 1\left(x_{+}=\delta \cos (\theta) / 2+\left(1-\delta^{2} / 4\right)^{1 / 2} \sin (\theta)\right)$. The dashed line (green) corresponds to the abscissa of the left endpoint of E1 $\left(x_{-}=\left|\delta \cos (\theta) / 2-\left(1-\delta^{2} / 4\right)^{1 / 2} \sin (\theta)\right|\right)$. 


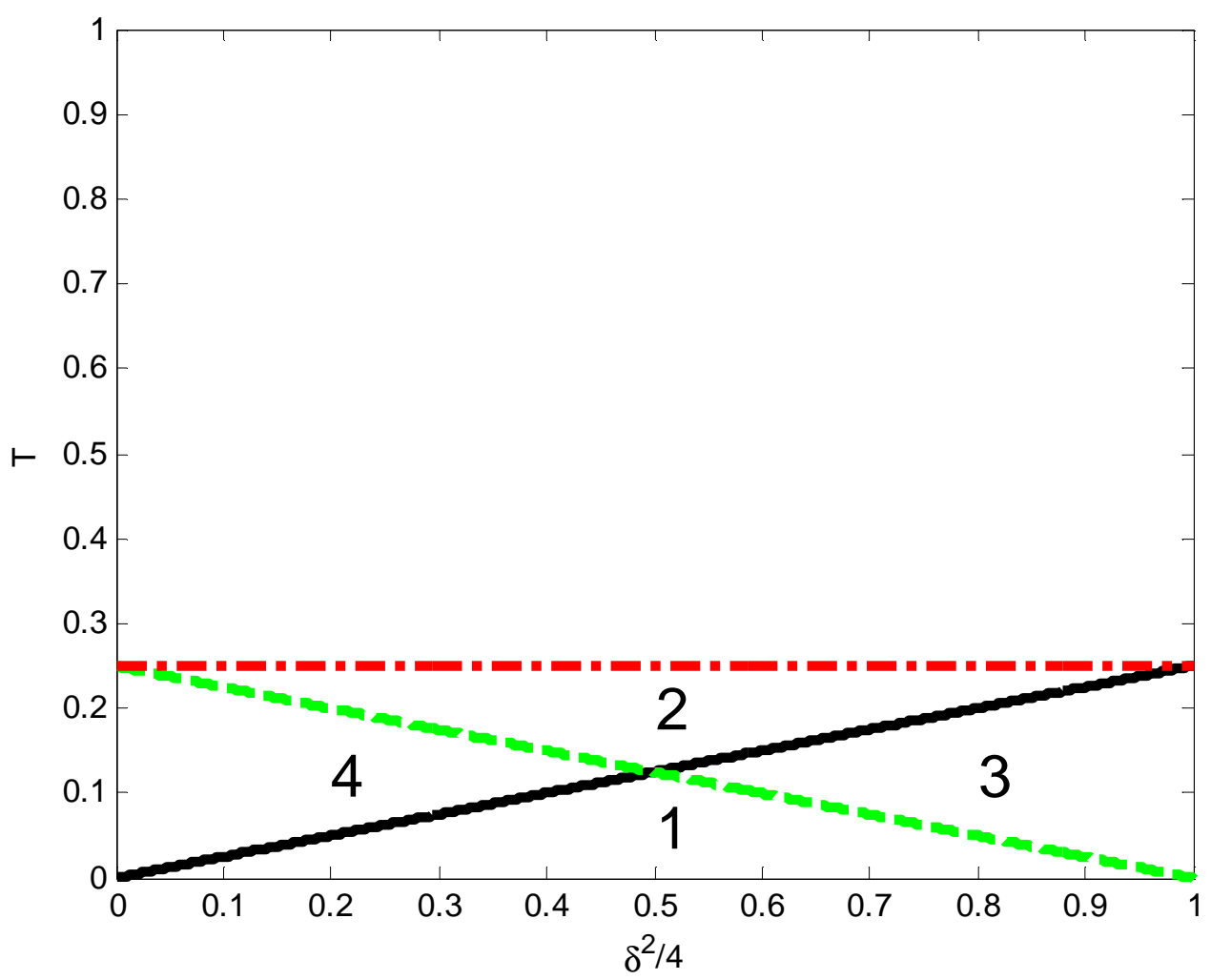

Figure 9: different areas of the $\left(\delta^{2} / 4, T\right)$ sub-space for a chord in a sphere (crossing the junction). The equations corresponding to the curves separating the different areas are:

Dashed line (green): $T=\left(1-\delta^{2} / 4\right) / 4$; dash-dot line (red): $T=1 / 4$; solid line (black) $T=\frac{1}{4} \frac{\delta^{2}}{4}$ 


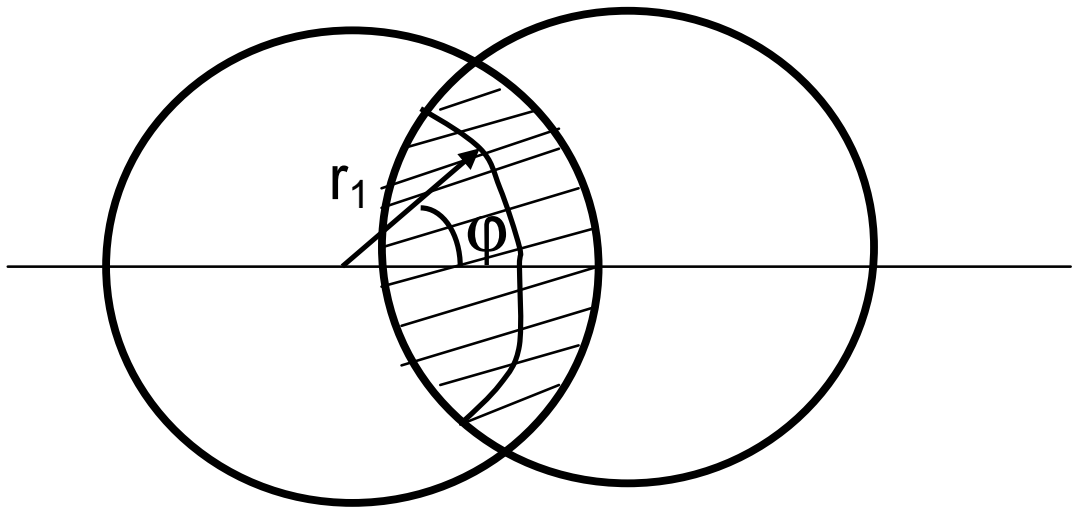

Figure 10: the dashed area (projection plane) is $C 1 \cap C 2$. 


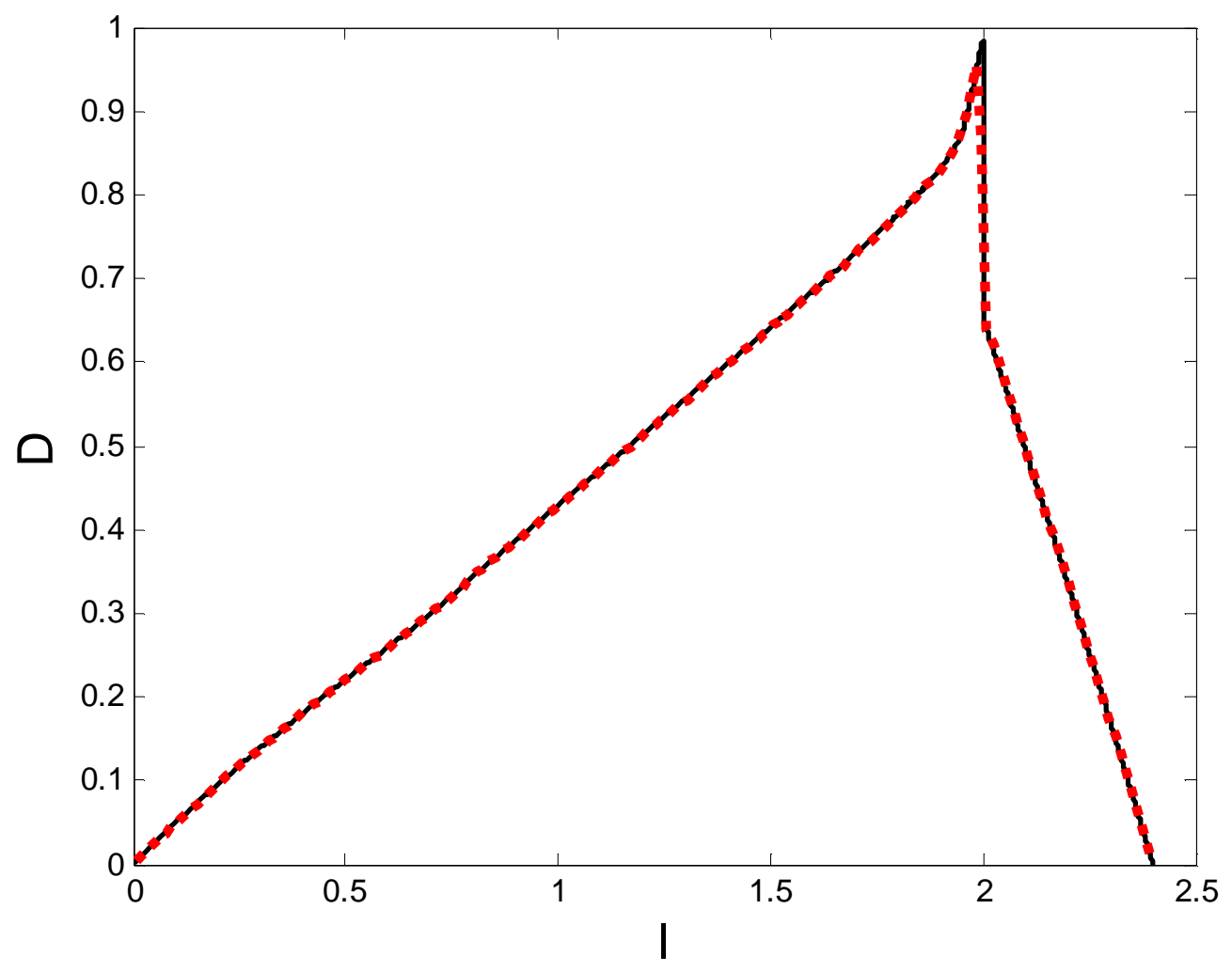

Figure 11: CLD (MCD): comparison between Monte Carlo simulations (dotted line; red) and analytical calculation (solid line; black). $\delta=0.4$. 


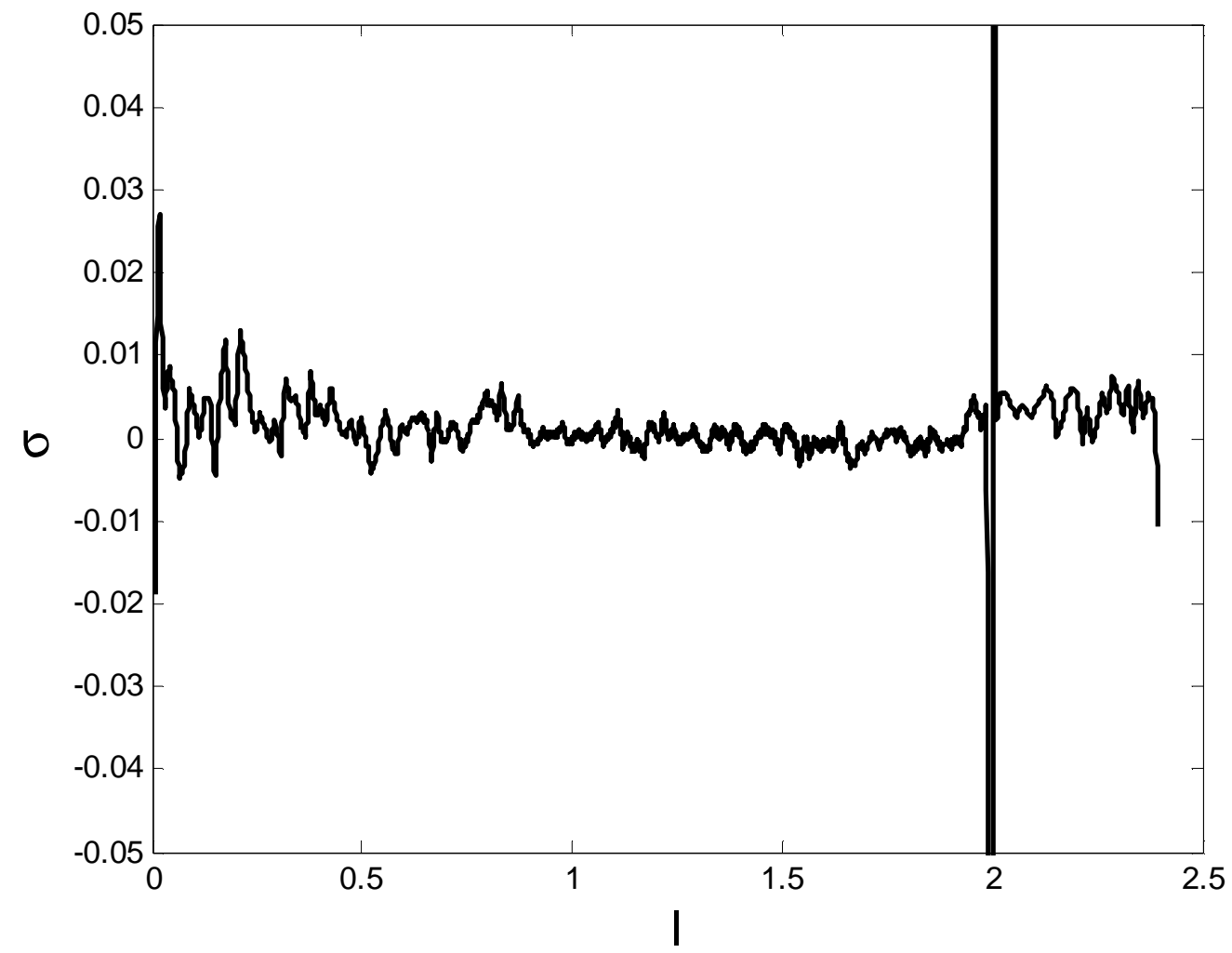

Figure 12: relative deviation between the CLD (MCD) calculated from Monte Carlo simulations and the analytical method (section 2). $\delta=0.4$. 